\title{
Wind Energy Contribution to a Sustainable Transport: The Case of Spain
}

\author{
Ángel Pérez-Navarro*, Fernando Ibáñez ${ }^{\circ}$, Carlos Sánchezº \\ Isidoro Segura ${ }^{\circ}$, and Carlos Álvarez ${ }^{\circ}$
}

Transportation accounts for almost one third of the total final energy consumption of Spain. This energy demand is almost completely met by oil, which in the case of this country, with almost no oil sources, must be imported. Meanwhile, wind energy is becoming an important component in the country's electricity generation portfolio, with a four fold increase in the last 5 years, going up to $8 \mathrm{GW}$ installed power in 2004 and a planned upgrading to $20 \mathrm{MW}$ predicted for 2010. So in a medium term scenario, this wind energy will become an important component, bigger than 30\%, in the country's electricity power generation system. Therefore, full exploitation of wind energy for transportation should be, from an economical point of view, a must. The two above-mentioned problems: coverage of transportation energy needs and full use of wind park capabilities, together with a third one related to the environmental impact of the use of oil in such a big quantity, could be solved if a hydrogen economy is successfully applied in the transportation sector. The critical questions are: (1) how much electricity would be required to make this solution possible and (2) by what scenario can surplus wind energy be best used to meet transportation energy needs, at least partly. In this paper, different scenarios for the use of the electricity generated in the wind parks for hydrogen production are proposed, deducing for each one the fraction on oil requirements that can be removed and the technical and economical viability of such scenarios in order to reach a sustainable transport in Spain.

Keywords: Transportation, Energy Needs, Wind Energy, Electricity, Hydrogen

\section{INTRODUCTION}

Transportation in Spain is based on the use of oil as can be deduced from Figure 1, where energy consumption structure for Spain in 2004 is deduced using data from IEA [1]. In the diagram, we have explicitly shown the electricity and hydrogen components given the important role to play by these two sources as energy vectors for future sustainable scenarios.

Total demand for primary energy in Spain during year 2004 was 142.4 Mtoe, from which 70.8 Mtoe where covered with oil. An important consumer for this big share of oil was transportation that demanded 38.5 Mtoe, almost $50 \%$ of the total oil consumption. In fact,

*Lead Author

Instituto de Ingeniería Energética

Universidad Politécnica de Valencia

Camino de Vera, 22 Valencia 46022 Spain

Phone: 34-963877270

Fax: 34-963877272

`Instituto de Ingeniería Energética Universidad Politécnica de Valencia

Camino de Vera, 22 Valencia 46022 Spain

Phone: 34-963877270

Fax: 34-963877272

(C) 2008 WEV Journal transportation accounts for almost one third of the total final energy consumption, with almost complete coverage by oil that, in the case of Spain with almost no oil sources, must be imported.

To avoid this big dependence on oil imports and also to remediate the environmental impact of this scheme of energy generation, Spain is developing an ambitious programme in renewable energy sources [2], especially in wind energy that is becoming an important component in the electricity generation scenario, with a four fold increase in the last 5 years up to reach $8 \mathrm{GW}$ installed power level in 2004 and a planned upgrading to 20 MW predicted for 2010, as shown at Figure 2. So in a medium term scenario, this energy source will become an important component, bigger than 30\%, in the Spain's energy portfolio and its full exploitation should be, from an economical point of view, a must.

Hydrogen is now considered as a serious candidate to become an important future energy vector [3]. Hydrogen, together with electricity, could cover a substantial fraction of the energy demand of several sectors of the economy including industry, residential, commercial and transport. By allowing a big penetration of distributed energy systems [4], in special renewable 
energies, and reducing the environmental impact of the energy sector it becomes possible to have a sustainable energy development and, in the case of Europe, dramatically reduce dependence on energy imports [5].

In this paper, we analyse different scenarios for the penetration of the hydrogen as an energy vector in the transportation sector, assuming that fuel cells and electric engines are available on an industrial basis for use in this sector. Particular attention will be given to generating hydrogen by using the overcapacity of the wind parks during hours of low demand from the electricity market. Section 2 studies the different scenarios estimating the amount of oil that can be reduced from the total consumption and the associated reduction in $\mathrm{CO} 2$ emissions. Section 3 analyses the technical viability of such scenarios and the requirements in each of them on electricity for the generation of the amount of hydrogen to supply. Finally, in section 4 we study the economical viability of the different scenarios.

\section{SCENARIOS FOR HYDROGEN PENETRATION IN TRANSPORTATION}

Prior to the study of hydrogen scenarios, and in order to facilitate the analysis of the improvement that they would provide, we have to deduce the tendencies for energy demand, oil consumption and CO2 emission in a business as usual (BAU) scenario where the country covers its energy needs without any drastic change in the structure of its energy system.
Figure 3 summarizes the data and results for the BAU predictions for Spain in the period 2005 to 2030. We are assuming the growth rates for gross national product and energy demand by the different sectors given at [6] and plot them at Figure 3a, together with the energy intensity that corresponds to these growth rates. It can be realized from the evolution of the energy intensity that a $42 \%$ improvement in energy efficiency and saving is obtained. Predictions for energy demand are plotted at Figure 3b: primary energy demand increases from 142 to 208 Mtoe, from which oil covers 70 and 103 Mtoe, respectively, and the transportation sectors demand for oil goes from 38.5 to 61.3 Mtoe. CO2 emission evolution is displayed at Figure 3c, they grow steadily from 355 in 2005 Mtons up to 511 Mtons in 2030. From those figures, oil consumption in transport is responsible for 77 and 122 Mtons, respectively, that represents an increase in the order of $60 \%$.

We have considered three different scenarios (Figure 4a) with different levels of penetration of hydrogen in substitution of oil in the transportation sector up to reach in 2030: 10, 25 and $50 \%$ of the total amount of the fuel needed in the sector, respectively. In addition a contribution from biofuels was assumed in all of them. This contribution evolves up to $10 \%$ in 2030 in accordance with the objectives of the UE commission directives. The growth rates for GNP and demand were the same that those assumed in BAU scenario.

Figure $4 \mathrm{~b}$ shows the evolution of hydrogen and oil demand to cover the transportation energy demand for the different scenarios under consideration. Scenario

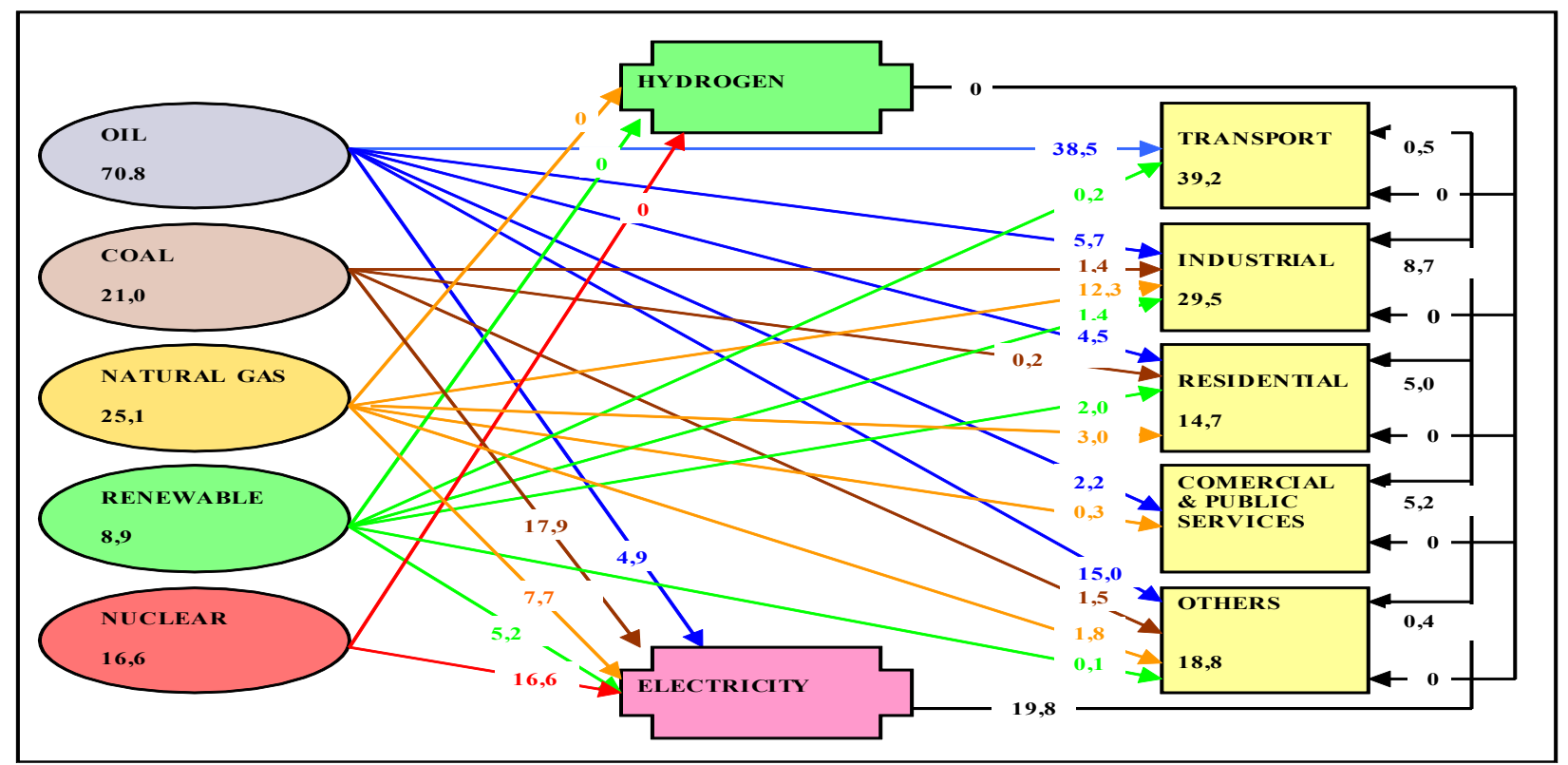

Figure 1: Spain energy consumption in 2004 (in Mtoe) 


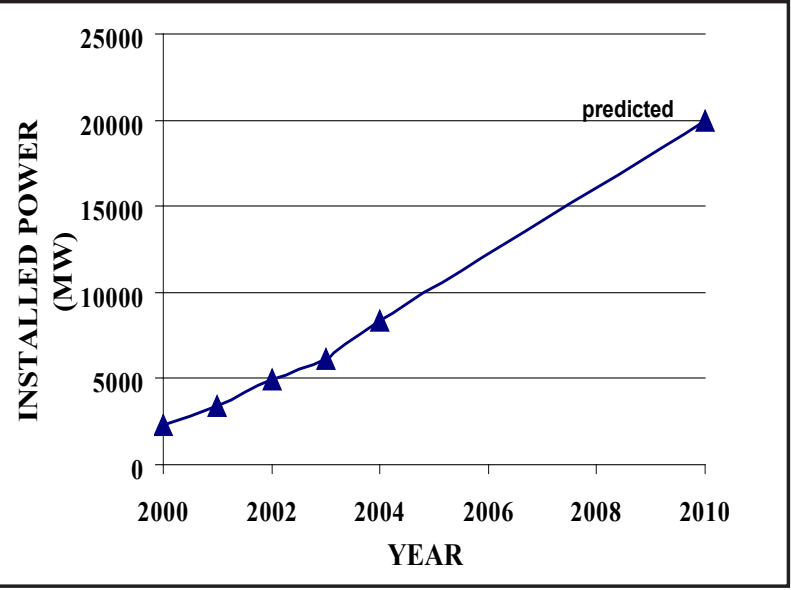

Figure 2: Evolution of installed wind power in Spain

H1 (10\% hydrogen contribution) implies a $28 \%$ increase in the demand of oil up to 50 Mtoe in 2030 and an amount of 6.6 Mtoe of hydrogen. Scenario H2 (25\% hydrogen contribution by 2030) enables to maintain almost constant in the level of 40 Mtoe the oil demand during the whole period of time and ask for 16 Mtoe of hydrogen by 2030. Finally, scenario H3 (50\% of hydrogen contribution in 2030) allows for a decrease of oil demand by a $36 \%$, reducing it to 25 Mtoe while asking for 31,5 Mtoe of hydrogen.

Figure 4c presents the simulations results for the evolution of the $\mathrm{CO} 2$ emissions in each of the three assumed scenarios. Obviously, H2 scenario allows for an almost constant emission level, while in scenario $\mathrm{H} 1$ the emissions increase by a $40 \%$ and in $\mathrm{H} 3$ they reduce a $36 \%$, going from 78 Mtons in 2005 to less than 50Mtons in 2030.

In conclusion, a scenario like $\mathrm{H} 2$ with a $25 \%$ of substitution of oil by hydrogen will be the minimum necessary to guarantee a sustainable transport, assuming that we can keep the supply of oil in Spain in the current levels. To reduce the external dependence in fuel supply and the $\mathrm{CO} 2$ emissions the hydrogen contribution should exceed that percentage in accordance with the goals imposed by the energy policy.

\section{TECHNICAL VIABILITY}

To determine the wind power needed to supply the electricity required in the electrolysis processes for hydrogen generation we are assuming an energy equivalence of 1 Mtoe $=11,90 \times 103 \mathrm{GWh}$, based on a hydrogen LHV of $120 \mathrm{MJ} / \mathrm{kg}$ and an equivalence of 2.8 Mtoe for each Mton of hydrogen.
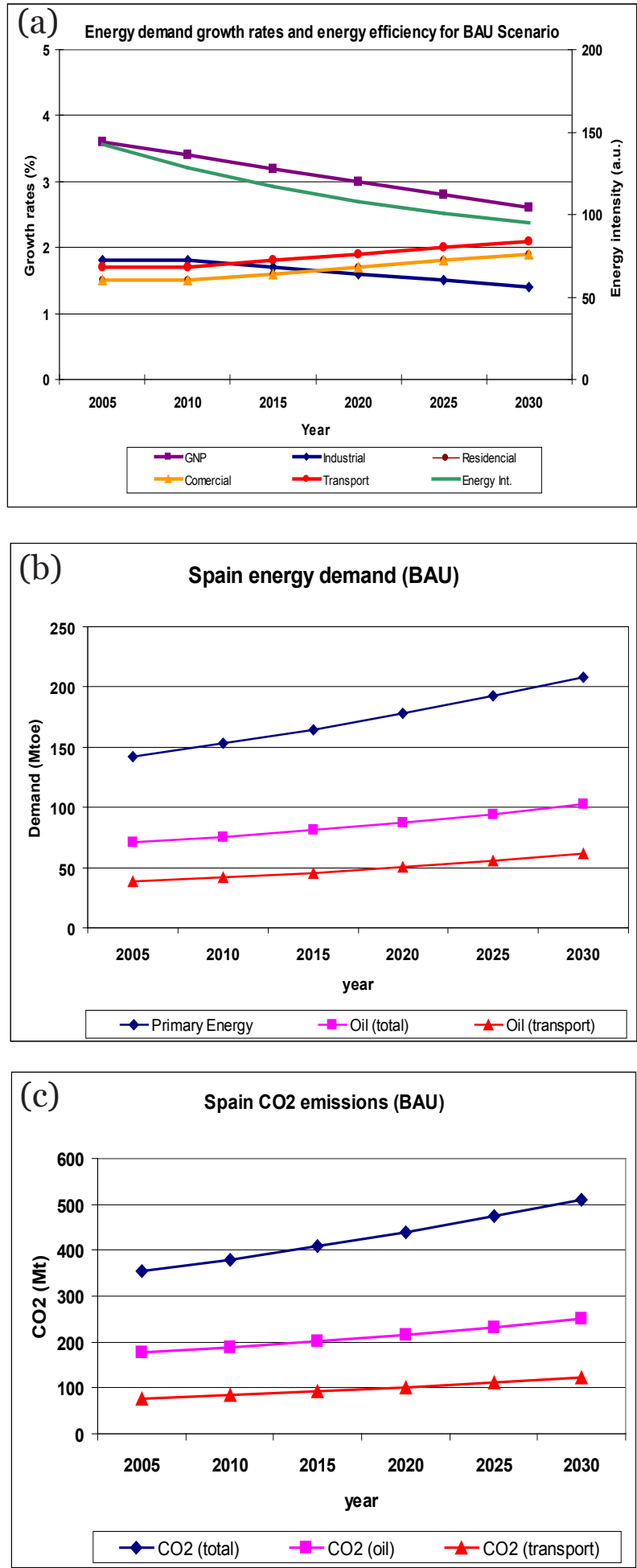

Figure 3: BAU scenario predictions a) growth rates, b) energy demand, c) $\mathrm{CO} 2$ emissions 

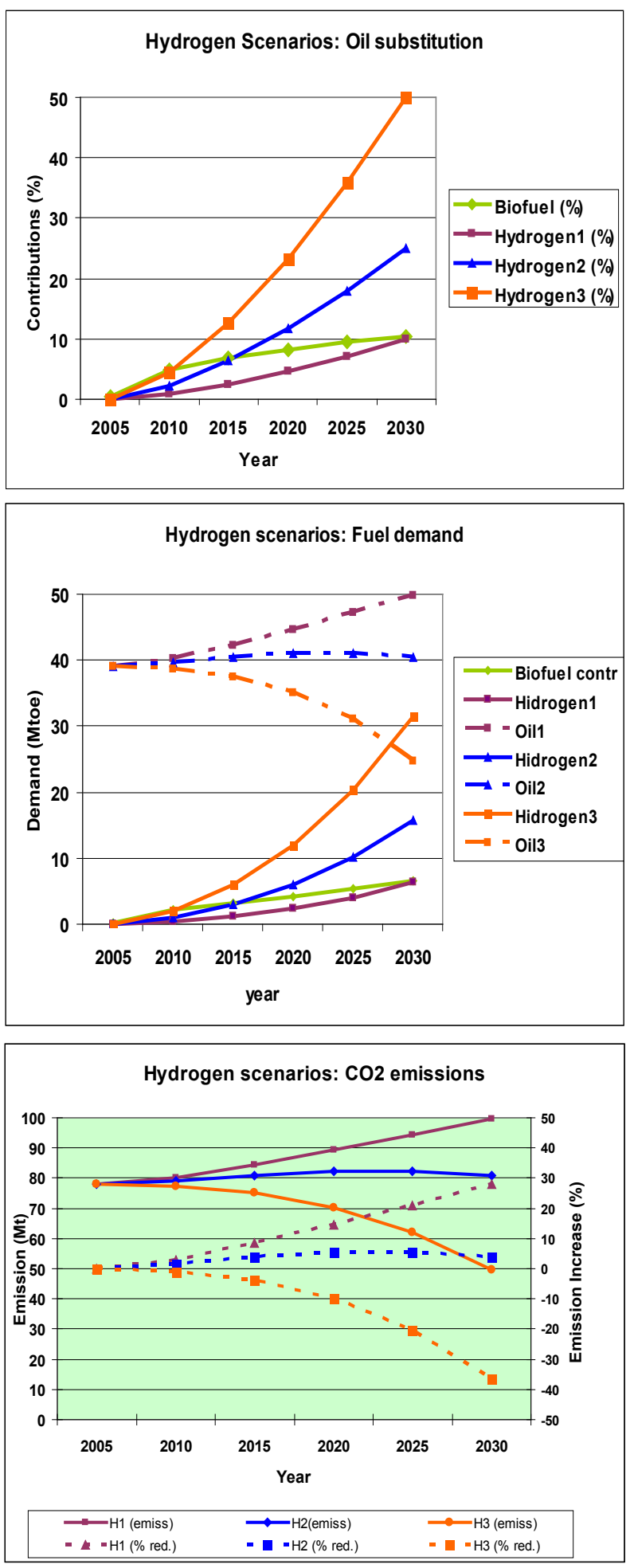

Figure 4: Hydrogen scenarios a) scenario contributions, b) fuel demand, c) $\mathrm{CO}_{2}$ emissions
The nominal wind power required to supply the total energy for the assumed scenario will be given by:

$$
\mathrm{P}_{\text {wind }}=\mathrm{E}_{\mathrm{H} 2} /\left(\mathrm{d}_{\mathrm{w}} \cdot \mathrm{t}_{\mathrm{w}} \cdot \eta_{\mathrm{el}} \cdot 365\right)
$$

Where:

$\mathrm{E}_{\mathrm{H} 2}$ is the energy required by the hydrogen

$\mathrm{d}_{\mathrm{w}}$ is the average time availability of the wind park (\%)

$\mathrm{t}_{\mathrm{w}}$ is the average period of time the wind power can be used for electrolysis (hours)

$\eta_{\mathrm{el}}$ is the electrolyser efficiency (\%)

Figure 5 shows the requirements in nominal wind power for the three scenarios studied in the previous paragraph assuming a $d_{w}=25 \%$ for the wind park operation; $\mathrm{t}_{\mathrm{w}}=24$ hours, full day dedication to hydrogen generation, and $\eta_{\mathrm{el}}=90 \%$, deduced from the simulations in [7] for an advanced alkaline electrolyzer, which operates at a pressure of 7 bar and at temperatures up to $80^{\circ} \mathrm{C}$. The cells are circular, bipolar, have a zero spacing geometry and consist of $\mathrm{NiO}$ diaphragms and activated electrodes. The system produces hydrogen from water electrolysis using electricity from the wind generator. Since the electrolyzer needs a DC current, the first stage in the hydrogen generator is a power electronics converter to supply it. The electrolyzer model is divided into three blocks in order to simulate thermal, electrochemical and thermodynamical aspects, and it can be used to predict the cell voltage, hydrogen production, efficiencies and operating temperature. This model is suitable for transient simulations; therefore it is compatible with these studies on wind energy storage that, given the wind fluctuation, has this stochastic character.

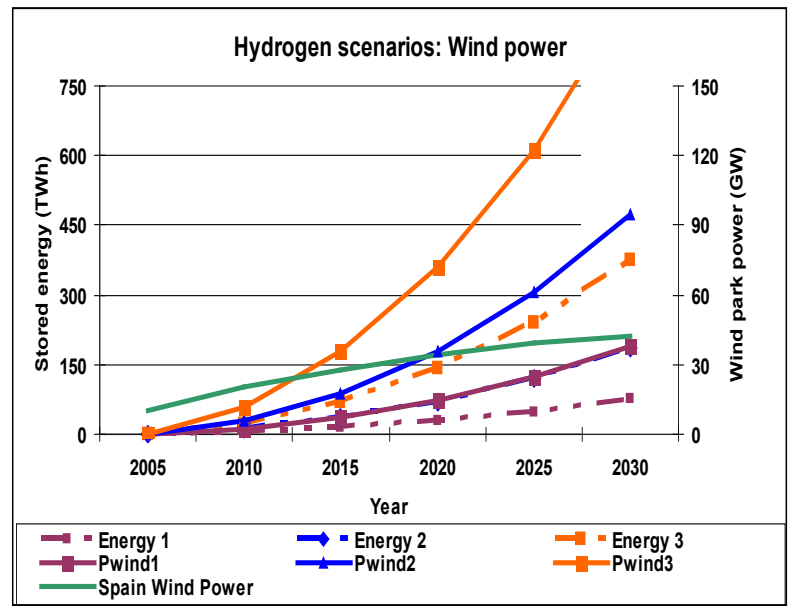

Figure 5: Wind power requirements for hydrogen scenarios 
According to these results, scenario $\mathrm{H} 2$ with a $25 \%$ penetration of hydrogen in the transportation sector that allows for the stabilization of the oil demand and consequently the CO2 emission at the level of 2005 will require a nominal wind power in the order of $90 \mathrm{GW}$. The programmed wind power to install in Spain is also plot in Figure 5, where a slow increase up to reach 42 GW in 2030 is assumed. So under these assumptions wind power will only cover half of the needs for hydrogen generation in a sustainable transportation scenario. This contribution will even decrease to $30 \%$ if we assume that the sharing with electricity demand from the grid during peak hours will take 8 hours per day. So, an increase in wind parks or use of other electricity sources is required to make these sustainable scenarios possible.

\section{ECONOMICAL VIABILITY}

Economical studies have been addressed to determine the period of time required for a profitable wind farm exploitation when it is partial or fully devoted to hydrogen generation. Following a methodology similar to that developed in [7] to analyze the economical viability of a backup system based on hydrogen to guarantee the availability of wind park generation, a basic scenario assuming a wind farm composed by 50 turbines of $1 \mathrm{MW}$ of power each one has been considered. The system generates electricity to inject in the grid during the peak demand hours and to produces hydrogen during the valley demand periods that, to simplify, we have assumed, respectively, to be the day and night periods. Economic income would be the cost of the generated electricity injected in the system at market price, taking advantage of being in peak demand hours, and the additional income from the produced hydrogen by using the electricity generated in the wind park to supply the electrolyser system during the demand valley hours.

Figure 6 plots the annual incomes coming from the selling of electricity and hydrogen generated by this $50 \mathrm{MW}$ wind park and the paying-off period for the installation when the assumptions displayed at Table 1 are made. The hydrogen market price has been fixed by making it equivalent to a cost of the oil barrel of $50 €$.

Results prove that income from hydrogen generation during low demand hours are lower, but in the same order than magnitude, than income from the sale of electricity during high demand peaks. The pay-off period for the capital cost of the park is about ten years in this scheme, that is two years longer than in the case of the park only dedicated to electricity production, assuming in this case sale at fixed distributor rate, as be seen at Figure 7, where we have included the simulation results

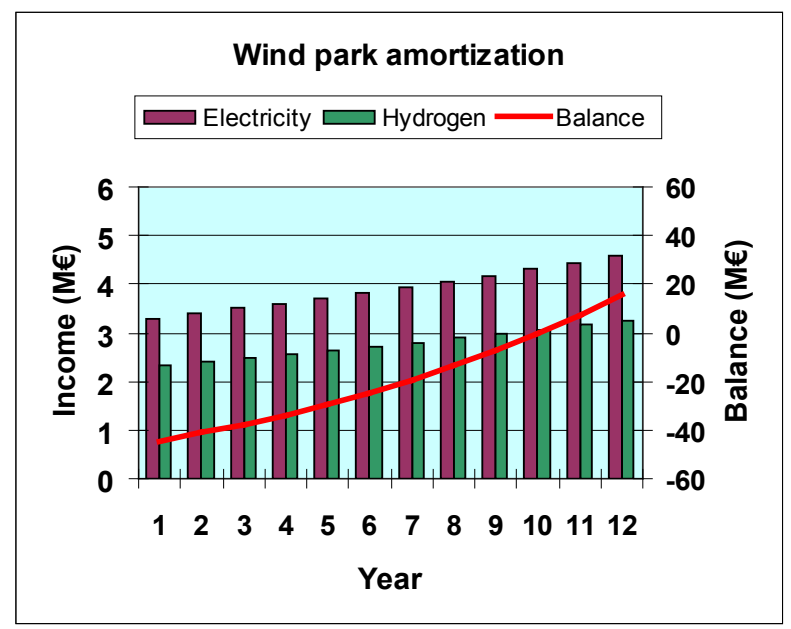

Figure 6: 50 MW wind park amortization

\begin{tabular}{|l|r|}
\hline Wind park nominal power (MW) & 50 \\
\hline Effective fraction of annual operation $(\%)$ & 25 \\
\hline Estimated installation cost range $(\mathrm{k€} / \mathrm{kW})$ & 2 \\
\hline Annual discount rate $(\%)$ & 6 \\
\hline Electricity market price $(\mathrm{c} € / \mathrm{kWh})$ & 90,448 \\
\hline Electricity distributor price $(\mathrm{c} / \mathrm{kWh})$ & 6,534 \\
\hline Hydrogen market price $(€ / \mathrm{kg})$ & 1,19 \\
\hline Annual increase in energy price $(\%)$ & 3 \\
\hline
\end{tabular}

Table 1: Assumptions for economical viability analysis

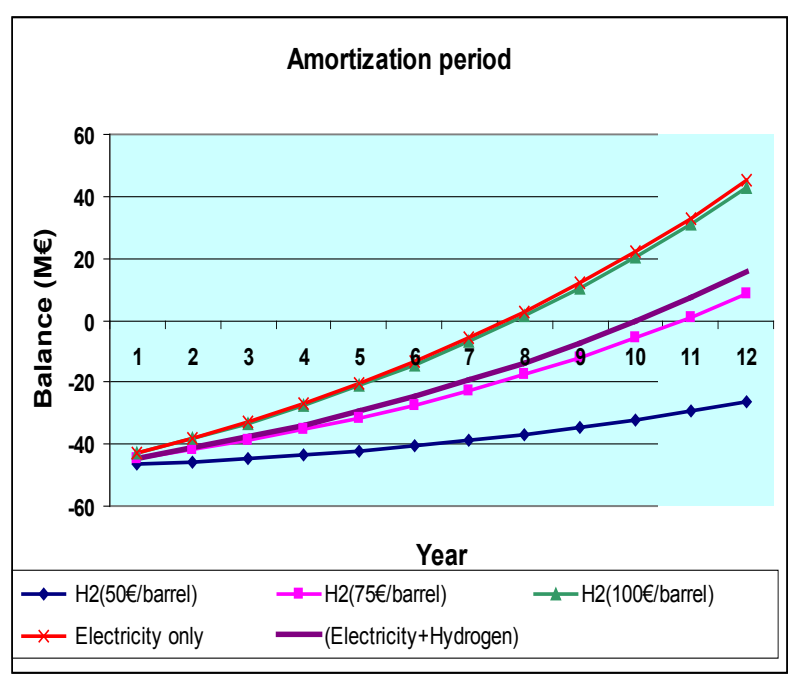

Figure 7: Pay-off periods for different scenarios 
for the dedication of the park exclusively to generate hydrogen for transportation assuming different cost for hydrogen equivalent to 50, 75 and $100 \$ €$ per oil barrel. It can be concluded this exclusive dedication of the park to hydrogen is fully competitive in the case of a $100 € /$ barrel scenario.

\section{CONCLUSIONS}

It is possible to stabilize the consumption of oil and the corresponding $\mathrm{CO} 2$ emissions in the transportation sector of Spain by a partial substitution with hydrogen, in the order of $25 \%$ of the total consumption. The electricity demand for the generation of the required amount of hydrogen would required a nominal wind energy power of about $90 \mathrm{GW}$, fully devoted to this task. To be economically competitive a 100 €/barrel scenario should be assumed. A more conservative approach would assume the coverage of the about one third of the hydrogen needs produced by wind park in a mixed operation mode, injecting electricity in the system during peak demand hours and using it for hydrogen production during valley demand periods. With this approach, a full use of the capabilities of the installed wind park in Spain will be guaranteed.

\section{REFERENCES}

[1] International Energy Agency. IEA Statistics. http:// www.iea.org/Textbase/stats/index.asp

[2] IDAE. 2005-2010. Spain Renewable Energies Plan. www.idae.es. 2005.

[3] S.S. Penner. Steps toward the hydrogen economy. Energy vol. 31, pp. 33-43, 2006.

[4] EU Directorate-General for Research. New Era for Electricity in Europe: Distributed Generation. Key Issues, Challenges and Proposed Solutions. EUR 20901. 2003/

[5] Commission of the European Communities. A European Strategy for Sustainable, Competitive and Secure Energy. COM 105. 2006.

[6] USA DoE. International Energy Outlook 2006 . DOE/EIA-0484. 2006.

[7] I. Segura et al. Technical requirements for economical viability of electricity generation in stabilized wind parks. To be published in International Journal of Hydrogen. Available online at www.sciencedirect.com. 2007. 